\title{
Experimental Art with Brain Controlled Interface
}

\author{
Tania Fraga ${ }^{1}$, Mauro Pichiliani ${ }^{2}$, and Donizetti Louro ${ }^{1,3}$ \\ ${ }^{1}$ IMA - Instituto de Matemática e Arte de São Paulo, São Paulo, Brazil \\ tania.fraga@gmail.com \\ ${ }^{2}$ Department of Computer Science, Instituto Tecnológico de Aeronáutica \\ São José dos Campos, Brazil \\ pichilia@ita.br \\ ${ }^{3}$ Department of Computer Science and History of Science, \\ Pontifícia Universidade Católica de São Paulo, São Paulo, Brazil \\ dflouro@pucsp.br
}

\begin{abstract}
This essay presents experimental computer artworks using Brain Controlled Interface (BCI). It points to a preliminary contextualization and general development emphasizing affective, sensory, poetic and aesthetic experiences intermediated by mindware devices. BCI offers a new research art field using a low-cost neuro system to explore human mind's untapped potential. A BCI for a Java3D framework allowed to arrive at the concept of exoendogenous interactivity. The main contribution of this essay is the novel use of affective quantified data to provide emotional feedback to computers and participants while experimenting an art piece, intertwining human affective states with computational autonomous processes. May one say that computer agents, by capturing world percepts, perceive the human mind activity? Possible answers to this question may open poetic and aesthetic research fields for artists, leading to a better understanding of how computers collect and respond to emotional states within human minds.
\end{abstract}

Keywords: Brazilian Computer Art, Brain Controlled Interface (BCI), exoendogenous interactivity, Caracolomobile, affective computing, awareness widgets, Emotiv neural EEG headset, machine percepts.

\section{Introduction}

Brain Controlled Interfaces (BCI) offers a new research field for artists. It allows them to glimpse at human mental capabilities using a low-cost off-the-shelf affective neuro system to interface with computers in order to explore and experiment with human mind's untapped potential. The experiments related here point to the poetic and aesthetic computing possibilities of BCI for the creation of artworks. Since sensations, perceptions and emotions play the most important role in any artwork and few research efforts are exploring the potential of BCI within the art domain, a proof-ofconcept was conceived. It is an artistic experiment pointing to some inquiries that have been gathered, which aim to investigate possible uses of affective interfaces for experimental artworks. Artists create metaphors clustering hypotheses, conjectures and intuitive perceptions, without the intention of demonstrating them as scientists do. 
Since there are not many artists experimenting with such interfaces just a few are quoted here. Some important research institutions focusing on similar issues in other domains were added. The rest of the paper is organized as follows: Section 2 describes a few artworks and concepts related with Human Computer Interface (HCI) and Brain Controlled Interface (BCI); It describes a first artwork created by Fraga in 2010, the robot 'Caracolomobile' [7]. This robot uses a BCI interface to control its expressive movements and sounds; Section 3 presents a novel possibility for artworks developing the concept of exoendogenous interactivity with the use of BCIs to capture emotions directly from the brain. These emotions are outputted to interfere in endogenous autonomous processes happening within the computer environment; Section 4 discusses the affective interface being investigated; finally, Section 5 presents the conclusions and possible developments for future artworks.

\section{Related Work}

\subsection{Human Computer Interface (HCI) and Exoendogenous Interactivity}

The interactivity in digital devices reveals advances over all collective imagination. The physical universe contains objects from the real world which are translated by mathematics, art and computer codes. What one is able to perceive are reflections of the world filtered through perceptive and cognitive systems, as stated by Claude Shannon and Charles Sanders Peirce. Therefore, one concludes that any worldview can be attributed to a set of relations, visual perceptions, and languages integrated as systems of values and beliefs that underlie culture. Larry Manevitz points to the fact one is working with mathematical neuroscience with applications being developed and applied during the recent advances in brain science.

The advances in mathematics and art through computer technology present a scenario of transformations ever experienced in the history of mankind. In a short time the technology changed ergonomic perceptual, sensory and cognitive behaviors, among others. To explore a Brain Controlled Interface (BCI) we have to establish a direct functional interaction between a human or animal brain and an external device [1]. There are numerous recent advances in BCI development and implementation driven by scientific and technological achievements. BCIs can be applied to assisting, augmenting, or repairing human cognitive or sensory-motor functions. Recent technological advances allow real-time on-line processing of multi-channel EEG data. The BCI also is called a mind-machine interface (MMI), or direct neural interface, or a brain-machine interface (BMI).

The French art professor Edmund Couchot defines interactivity in two ways: exogenous and endogenous interactivity [3]. Exogenous interactivity happens when the interactor's ${ }^{1}$ act over the artworks domain whose environments are programmed to answer for their actions, transforming them. Within endogenous interactivity environments there are virtual or physical autonomous agents who interact with one

\footnotetext{
${ }^{1}$ The word interactor was created by the Brazilian theorist and art critic Arlindo Machado to refer to the participant's actions within an interactive computer art experience.
} 
another and with the setting following a diverse set of rules and algorithms independently from the interactor's actions. In what Fraga has defined as exoendogenous interactivity the interactors affect either the state or the behavior of these autonomous agents. These aspects will be better developed at sections 3 and 4 .

\subsection{Brain Computer Interface (BCI) in Experimental Artworks}

When one refers to systems, specifically in brain-computer relationship, one understands the processes that are embedded in their architecture. This understanding leads to late nineteenth century discoveries by Willem Einthoven (1860-1927), Dutch physician, who contributed to the advancement of Science in electrophysiology, culminating in the development of the electrocardiograph. The successors of Einthoven advanced the coronary and angina syndromes theory, among other medical theories of great importance in the history of medicine. On the other hand, there is also the excitement when discussing the theory of models of later artificial intelligence applications, unprecedented in the history of applied sciences.

The history of BCIs started with Hans Berger's discovery, in 1924, of the electrical activity of the human brain and the development of electroencephalography (EEG). Following Berge's discoveries, the first BCI was described by Dr. Walter Grey, in 1964. After the 70's, computing, electronics and sensors technology allowed the development of external devices and algorithms capable of simulating the motor cortex firing patterns of neurons with their specific muscular responses. Actual researches in nanotechnology, biomaterials, and humans central nervous systems (CNS) are allowing the commercialization of BCI affordable devices. As stated before, BCI is a new research field for artists, which allows one to explore and experiment the human mind's potential glimpsing at its mental capabilities.

There are not many artists experimenting with such interfaces. Among them one may quote the Canadian Sandra $\mathrm{K}^{2}$ that proposes to draw with brain waves and the musician Eduardo R. Miranda of the University of Plymouth, UK, that researches a BCI "that can detect and recognize musical ideas in the minds of composers with up to 99 percent accuracy" [10]. These researches may be applied in games. BCI gaming is a recent field and most games are still in the proof-of-concept stage [16]. It is important to say that in 2006, Paul Fishwick at MIT, pointed to the aesthetic computing possibilities of BCIs [6] and many important institutions are developing BCI research in other domains. Although they do not focus on art they are relevant to the field. Bellow we quote just a few of them such as: The MaxPlanck Institute of Intelligent Systems ${ }^{3}$ [19]; the MIT [9] and Microsoft [18].

2 See: http://neurofuture.blogspot.com.br/2006/10/ brain-computer-interface-drawing.html

${ }^{3}$ The MaxPlanck Institute of Intelligent Systems points to Brain-Computer Interfaces (BCIs) as mindware enabling "subjects to communicate by intentionally modulating their brain's electromagnetic field. This field can be recorded outside the brain by methodologies such as EEG, MEG, or ECoG, analyzed with the aid of machine-learning algorithms, and then translated into commands that may be used to control computers or neuroprostheses". 
In Brazil the computer artist and researcher Tania Fraga, one of the authors of this paper, is working with BCI interfaces to develop artworks. In 2010, she created the robot 'Caracolomobile'4 [7] (Figure 1) and is now developing the virtual reality environment, 'NumericVariations' (work in progress, Figures 3, 4, and 5), in collaboration with Pichiliani and Louro.

This whole quest was begun in 2007 when Fraga had the opportunity to try the NeuroSky headset [11] prototype at Yuri's night at NASA Ames Research Centre. After this mind blowing experiment many questions have arisen. By brainstorming the aesthetic and poetic possibilities that have been opened up by such an approach the authors have come up with a set of questions:

- Would it be possible to generate physical objects movements with any kind of mental process without any invasive procedure?

- Has a focused thought the ability to affect expressively a virtual environment or a physical mechanical system or object?

- May a consciously controlled affective state provoke expressive changes in the dynamic of an autonomous computer process?

- Would an unconscious affective state affect the dynamic of the virtual environment in any expressive way?

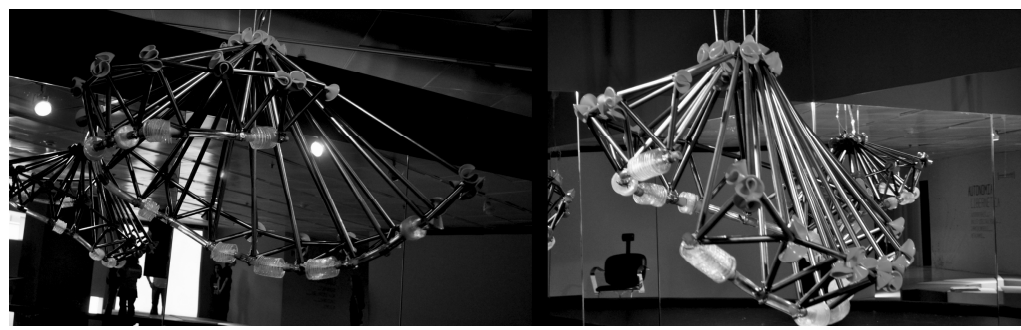

Fig. 1. The robot 'Caracolomobile'

In 2010 Fraga received an award from the Brazilian Itau Cultural Institute to build an experimental artwork, a pneumatic robot, 'Caracolomobile', for the Art and Technology biennale, 'Art.ficial Emotion 5.0' [4]. Therefore it was time to look for answers for some of the questions formulated above and the robot 'Caracolomobile'

4 The robot is a responsive object made of anodized blue titanium with flexible red silicon and polyurethane details. It is controlled by a pneumatic system related with an affective system that allows it to present expressions through sounds and movements. The robot 'perceives' the interactor's affective states expressing itself in answer to these stimuli. A Java interface (Emotiv-J) allows the robot to identify the interactor's emotional states captured through the Emotiv neural headset, a not intrusive device, which reads the mind fluctuations. This headset has 16 neural sensors able to articulate expressive, affective and cognitive states. The neural headset communicates the interactor's affective and expressive states to the robot's recognition system. The system identifies these states and translates them into images, sounds and movements. See [2]. 


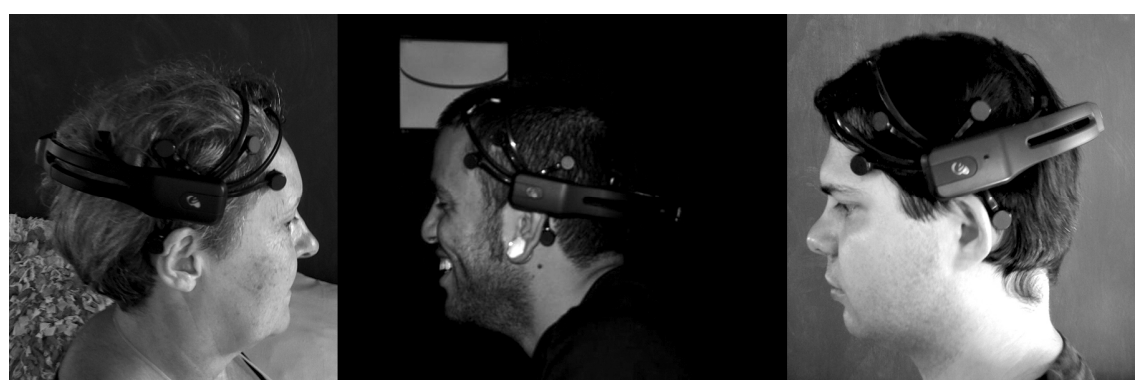

Fig. 2. Different interactors wearing the Emotiv headset device

was built. It answers expressively with movements and sounds to affective and expressive human states. The robot acts in symbiosis ${ }^{5}$ with the interactor using a graphic interface to monitor these affective and expressive states captured by the Emotiv neural headset [5] (Figure 2).

The Emotiv neural headset was developed by a multidisciplinary international scientific team among whom we may quote the awarded Director of the Center for the Mind from Sydney University, Allan Snyder, and Neil Weste, renowned inventor, one of the authors of the CMOS system, who also developed projects at Bell Labs, Cisco Systems and many universities [20].

According to Theodore W. Berger, BCIs may be classified into two categories, "depending on the placement of the electrodes used to detect and measure neurons firing in the brain. In invasive systems, electrodes are inserted directly into brain tissue. In non-invasive systems, electrodes are placed on the scalp and use electroencephalography (EEG) or electrocorticography (ECoG) to detect neuron activity. (...) Other sensing methods employed in BCI systems in an auxiliary capacity include magneto encephalography (MEG), thermography, functional magnetic resonance imagery (fMRI) interpretation, and analysis of near infrared spectrum (NIRS) activity" [1].

In 'Caracolomobile' the headset's sixteen neuro biometric sensors capture the interactor's mind fluctuations and transmit them to the robot that answers them with sounds and movements. Built with titanium tubes, shaped as a snail shell, 'Caracolomobile' was hanged over an artificial water mirror surrounded by mirrors showing itself at many angles. The robot opens itself producing low pitch sounds when the interactor is relaxed. It closes emitting high pitch, almost hysterical, sounds when she/he is stressed. It was designed and built in modules: a pneumatic module, an electronic module and the BCI module [2].

Due to the short time available to develop the hardware and software (three months only) strict priorities had to be set up. Therefore, as stated above, it was chosen that the robot would answer to meditative and expressive affective states with pulsating

\footnotetext{
5 The definition of symbiosis that better fits the present context was proposed by Licklider quoted by Donald Norman. The proposed concept of symbiosis results from the "fusion of two components, human and machine, and the mixture is soft and fruitful, the resulting collaboration exceeding what anyone is capable of doing alone" [12].
} 
movements and sounds, opening and closing itself. The sounds were pre computed since there was no time to program a composing musical interface. The movements resulted from the interplay of three pneumatic actuators controlled by three valves and three electronic boards. ASC values were used to set up a control table with values related to the amount of air to be blown. Therefore it was possible to create a repertoire of possible movements to be chosen to express the mind fluctuations digitalized by the headset. During the three months of its development some basic movement patterns were created, but the expressiveness of such movements needs improvements. After the exhibition finished the research has continued and different sizes of pneumatic cylinders have been tried. After such experimentations it was possible to arrive at the conclusion that the main difficulties to achieve the desired fluidity of movements were due to the interplay of varied tensions and compressions in the dynamic spiral trelliswork structure. These problems are mechanical and are not due to the BCI, but by understanding them it has been possible to adapt the parameters aiming to achieve the desired aesthetic and poetic goals.

\section{Novel Possibilities for Artworks: Exoendogenous Interactivity}

In 2003, with a previous grant from Itau Cultural Institute, Fraga began to develop a Java 3D framework for virtual reality artworks. Since 2006 she has adapted a set of very simple algorithms ${ }^{6}$ allowing the development of endogenous artworks where autonomous processes happen within the computer environment. With Pichiliani and Louro's collaboration a much bolder approach was conceived arriving at the concept of exoendogenous interactivity, which will be better explained in the next section. This approach goes forward into the artistic search for poetic and aesthetic qualities entwined with the scientific search for logic and exactitude and the technical search for functionality.

\section{The Affective Interface}

Aiming to investigate the possibilities of an affective interface for the experimental artwork, 'NumericVariations' (work in progress), a proof-of-concept was conceived. It also explores the Emotiv low-cost off-the-shelf affective neuro system using it "to provide emotional awareness information" [15] for artwork systems.

The artwork's virtual reality environment is composed by six cyberworlds. Each two of them approach one different algorithm achieving opposite aesthetic and poetic results. These algorithms allow autonomous processes to develop within the computer environment. The BCI captures the interactor's affective states of calm/excitement and input them into the autonomous agents' behavior parameters. Therefore the virtual agents change their behaviors accordingly to these human emotions. The calm/excitement states are transcodified as positive values.

${ }^{6}$ These algorithms are: collision detection, flock and growth algorithms, and game of life algorithm. Only the three former ones are being used in 'NumericVariations'. 
The ranges of calm/excitement detected by the interface provide the output scores reflecting the interactor's affective changes over time periods. In cyberworlds 'Branqueza' and 'Negreza' (Figure 3) these scores are passed as velocities and size of spheres that autonomously collide with the 'ground'. For cyberworlds 'DancingBots_01' and 'DancingBots_02' (Figure 4) a flock of dancing bots ${ }^{7}$ were programmed to behave by seeking and escaping from one another. The scores are passed as values that allow the bots that seek, the seekers, to run more or less eagerly after the bots that escape, the escapees. Therefore they define the dynamic of the autonomous process happening. For the cyberworlds 'FractalGrowth_01' and 'FractalGrowth_02' (Figure 5) the scores are passed as the ageing time of the growing processes.
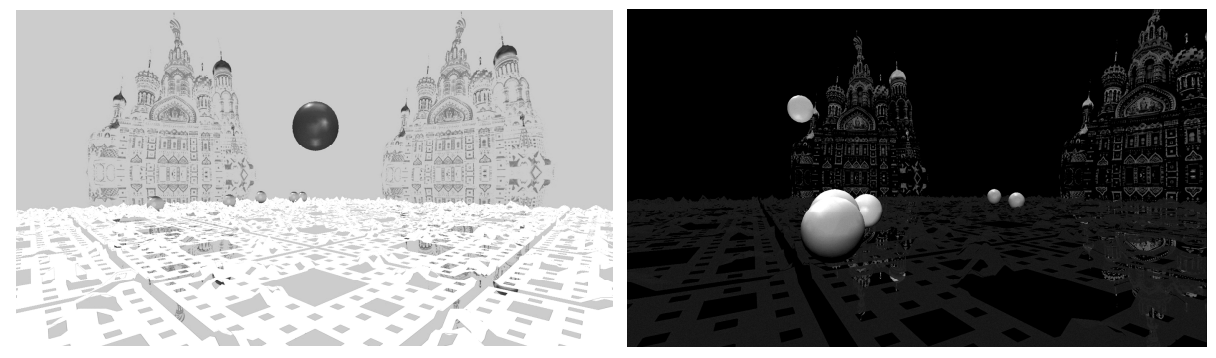

Fig. 3. 'Branqueza' (left) and 'Negreza' (right): virtual worlds with autonomous collision processes affected by human emotional states

"Emotions play an important role in human interaction, communication, coordination, and cooperation. (...). While there is a growing interest in providing computational support for recognition and representation of emotions, few research and artistic efforts explore the potential of BCI to collect, represent and explore emotional awareness (...). The study of emotions involves many disciplines such as neurology, psychology, and physiology. From the computer science point of view, the Affective Computing area explores how computing relates to, arises from, or deliberately influences emotions [14] arguing that by conferring the computers with abilities to recognize, model, and synthesize emotions, human-human and humancomputer interaction will be improved" [15].

By reflecting upon these experiments the necessity to have a better understanding of some of the perceptive processes involved arose. Due to the peculiar characteristics of these artworks Peirce's theory of perception ${ }^{8}$ [13] was chosen as the most adequate to reflect upon and to understand such processes. Therefore it is necessary to briefly point to Peirce's definition of percept. Percepts are reflections of the physical world. They go through the sensory system unveiling dimensions of the material things for all beings, including animal, plants and machines. Artists assemble experiments with

\footnotetext{
${ }^{7}$ Bots are virtual autonomous agents.

${ }^{8}$ To Peirce's theory of perception see Lucia Santaella [17].
} 

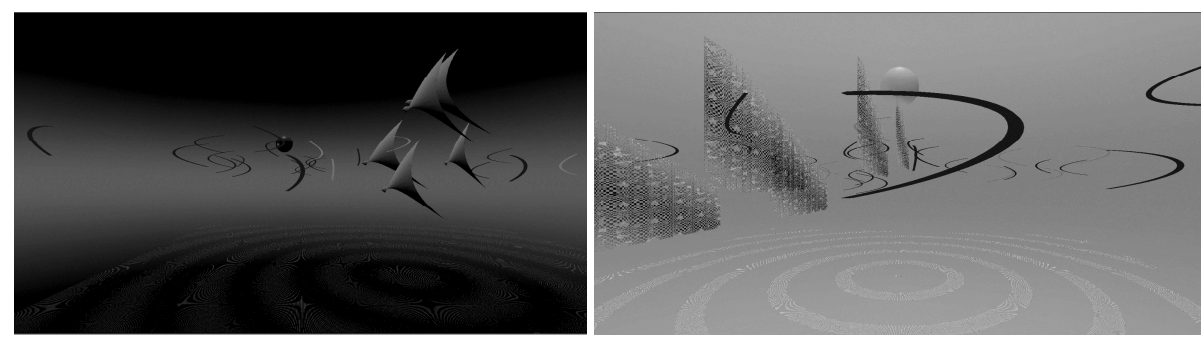

Fig. 4. 'DancingBots_01' (left) and 'DancingBots_02' (right): virtual worlds with autonomous flock behaviors affected by human emotional states

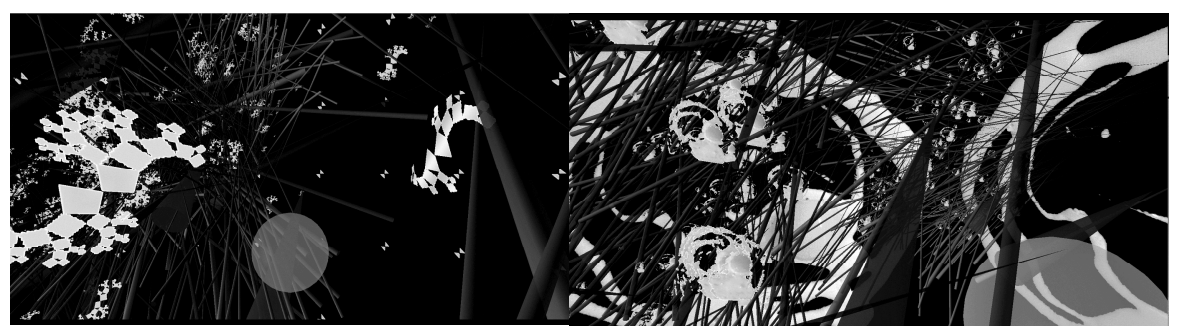

Fig. 5. 'FractalGrowth_01' (left) and 'FractalGrowth_02' (right): virtual worlds with autonomous growing processes affected by human emotional states

signs aiming to create actions or situations rich in percepts. Such percepts are characterized as signs, interdependent and plurals, among subject-sign-language and the object-thing. They spread over us "through all sensory pathways". They fly within ones perceptive processes being "absorbed by the nets of interpretative models" one is doted [17]. Within the machine's processes the percepts are analyzed and evaluated in function of the action one intends to achieve from the machine, adapting them for aesthetic and poetic purposes.

\section{$5 \quad$ Conclusions and Future Work}

In this essay, the exploration of Brain Controlled Interfaces (BCI), aiming to experiment with poetic and aesthetic possibilities, used a mindware to capture emotional data exploring them in artworks. The main contribution of this paper is the novel use of affective data obtained directly from the brain to provide emotional feedback to the public interacting with the art work.

The main question is to inquire about the related possibilities to intertwine human affective states with computer autonomous processes. May one say that the computer agents, by capturing world percepts, are perceiving the human mind activity? How they convey, interpret, and react to it? Possible answers to this question may open poetic and aesthetic research fields for artists, leading to a better understanding of how computers collect emotional states within human minds, applying brain sensing 
technologies for the art domain. Also, the exploration of this relation between the artwork and the interactor may reshape how one feels, behaves, understands and, ultimately, treats computer autonomous process after being aware of one's own emotions. The authors believe this approach represents an initial step in exploring a relevant area by applying it to Computer Art investigation.

To complement this approach for future works, one has to consider potential scientific applications of BCIs that are beyond visible controllers such as: motion control, medical devices, controlling home appliances, phone address books, haptic devices, web search engines, among others. Some mathematical and artworks may improve natural ideas through BCIs explorations for visual images generation. The IMA-SP [8] recent group works are demonstrating human ability to voluntarily regulate the activity of neurons responsible for visual images generation. The experiments were based on mathematical models and exploratory programming together with aesthetics through computer arts concepts. 'Ilustrativo' (Figure 6) is an example of this approach. In it EEG is recorded by electrodes placed on the scalp and digitized by an analogical to digital converter device (ADC). Computer process extracts features most suitable for identifying the subject's intentions. When intention is classified, a certain command is sent to an external device (e.g. a display). Feedback provides the subject with results of his/her actions thus allowing him/her to adapt to the system behavior.

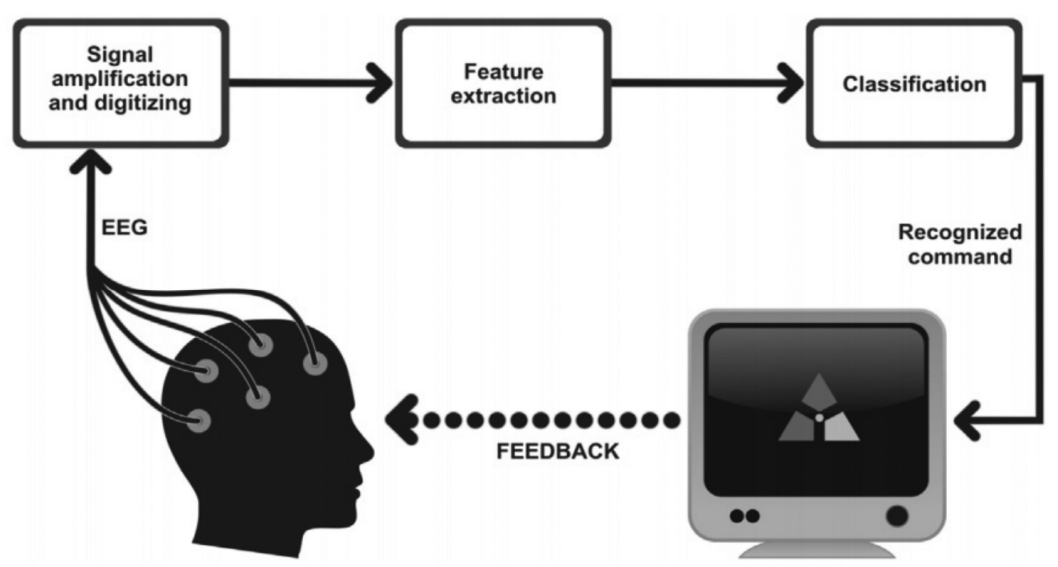

Fig. 6. General scheme of an EEG-based BCI [20]

Acknowledgements. The authors want to thank the São Paulo Research Foundation (FAPESP) and Itau Cultural Institute for the grants that allowed the experimentation of the concepts related above and the construction of the robot 'Caracolomobile'; and the School of Communication and Arts of the University of São Paulo (ECA-USP), the company Globalcode, and the many individuals involved in the initial exploration of the Emotiv EEG headset. 


\section{References}

1. Bobrov, P., Frolov, A., Cantor, C., Fedulova, I., Bakhnyan, M., Zhavoronkiv, A.: BrainComputer Interface Based on Generation of Visual Images. PLoS ONE 6 (2011)

2. Caracolomobile on Vimeo (2013), http: / / vimeo. com/15751832

3. Couchot, E.: A tecnologia na arte: da fotografia a realidade virtual. UFRGS Publisher, Porto Alegre (2003)

4. Emoção Art.ficial 5.0 (2013), http: / / www. emocaoartficial.org.br/en/ artistas-e-obras/emocao-5-0/

5. Emotiv EPOC Neuro heaset (2013), http://www. emotiv.com/store/hardware/epoc-bci/ epoc-neuroheadset/

6. Fishwick, P.A.: Aesthetic Computing. MIT Press (2008)

7. Fraga, T.: Caracolomobile: affect in computer systems. In: AI \& Society Journal: A Faustian Exchange, vol. 21, pp. 1-15. Springer, London (2012)

8. Institute of Mathematics and Arts from São Paulo (2013), http: / /www.ima-brazil.com/

9. Lotte, F., Langhenhove, A., Lamarche, F., Ernest, T., Renard, Y., Bruno Arnaldi, B., Lécuyer, A.: Exploring Large Virtual Environments by Thoughts Using a Brain Computer Interface Based on Motor Imagery and High-Level Commands. Presence: Teleoperators and Virtual Environments 19(1), 54-70 (2010)

10. Miranda, E.R.: Brain-computer music interface for composition and performance. Journal on Disability and Human Development 5(2), 119-126 (2011)

11. NeuroSky Neuro headset (2013), http: / / www . neurosky . com/

12. Norman, D.A.: The Design of Future Things. Basic Books, Philadelphia (2009)

13. Peirce, C.S.: Semiótica. Perspectiva, São Paulo (1990)

14. Picard, R.: Affective computing. MIT Press, Cambridge (2000)

15. Pichiliani, M.C., Hirata, C.M.: T., Fraga: Exploring a Brain Controlled Interface for Emotional Awareness. In: SBSC 2012 -Brazilian Symposium on Collaborative Systems, pp. 15-18 (2012)

16. Reuderink, B.: Games and Brain-Computer Interfaces: The State of the Art. CTIT technical report series, Netherlands (2008)

17. Santaella, L.: Percepção. Experimento Publisher, São Paulo (1993)

18. Tan, D.S., Nijholt, A.: Brain-computer interfaces. Springer, London (2010)

19. The MaxPlanck Institute for Intelligent Systems (2013), http: / / www.is.mpg.de/2178/en

20. Viveiros, E.R.: Mindware semiótico-comunicativo: aplicação didática no Ensino de Física para deficientes visuais utilizando uma interface cérebro computador. PhD Thesis at State University Júlio Mesquita, Baurú (2012) 